\title{
Existence of solutions for impulsive fractional evolution equations with periodic boundary condition
}

\section{Baolin Li* and Haide Gou}

"Correspondence:

ghdzxh@163.com

College of Mathematics and

Statistics, Northwest Normal

University, Lanzhou, 730070, P.R.

China

\begin{abstract}
This paper discusses the existence of mild solutions for a class of fractional impulsive evolution equation with periodic boundary condition and noncompact semigroup. By using some fixed point theorems, the existence theorems of mild solutions are obtained, our results are also more general than some well-known results. Furthermore, as applications that illustrate the abstract results, two examples are given.
\end{abstract}

MSC: 34K30; 34K45; 35B10; 47D06

Keywords: periodic boundary value problem; impulsive fractional evolution equation; $C_{0}$-semigroup

\section{Introduction}

Fractional differential equations arise in many engineering and scientific disciplines as the mathematical modeling of systems and processes in the fields of physics, chemistry, aerodynamics, electrodynamics of complex medium, polymer rheology, and they have emerged as an important area of investigation in the last few decades; see [1-5].

The theory of impulsive differential equations is a new and important branch of differential equation theory, which has an extensive physical, population dynamics, ecology, chemical, biological systems, and engineering background. Therefore, it has been an object of intensive investigation in recent years, some basic results on fractional impulsive differential equations have been obtained and applications to different areas have been considered by many authors, we refer to [6-9] and the references therein.

At present, the concept of solutions for impulsive fractional differential equations [221] has been argued extensively. There are some ways to consider the notion of solution of the form

$$
\begin{cases}{ }^{c} D_{t}^{\alpha} u(t)=f(t, u(t)), & t \in J^{\prime}:=J \backslash\left\{t_{1}, \ldots, t_{m}\right\}, J:=[0, T], \\ \left.\Delta u\right|_{t=t_{k}}=I_{k}\left(u\left(t_{k}\right)\right), \quad k=1,2, \ldots, m, & \\ u(0)=u_{0} . & \end{cases}
$$

One way is to change the lower limit to $t_{k}$ on each impulsive and consider (1.1) on $\left[t_{k}, t_{k+1}\right]$. This approach is used in several papers $[2-6,11]$. In this way, the problem (1.1) is rewritten

(c) The Author(s) 2017. This article is distributed under the terms of the Creative Commons Attribution 4.0 International License (http://creativecommons.org/licenses/by/4.0/), which permits unrestricted use, distribution, and reproduction in any medium, provided you give appropriate credit to the original author(s) and the source, provide a link to the Creative Commons license, and indicate if changes were made. 
as to the following integral equation:

$$
u(t)=\left\{\begin{array}{l}
u_{0}+\int_{0}^{t} \frac{(t-s)^{\alpha-1}}{\Gamma(\alpha)} f(s, u(s)) d s, \quad t \in\left[0, t_{1}\right), \\
\vdots \\
u_{0}+\int_{t_{k}}^{t} \frac{(t-s)^{\alpha-1}}{\Gamma(\alpha)} f(s, u(s)) d s+\sum_{0<t_{k}<t} \int_{t_{k-1}}^{t_{k}} \frac{\left(t_{k}-s\right)^{\alpha-1}}{\Gamma(\alpha)} f(s, u(s)) d s \\
\quad+\sum_{0<t_{k}<t} I_{k}\left(u\left(t_{k}^{-}\right)\right), \quad t \in\left(t_{k}, t_{k+1}\right], k=1,2, \ldots, m .
\end{array}\right.
$$

Taking another way, the work in $[7-10,12,13,15-19,21-23]$ is based on the result that problem (1.1) is equivalent to the following integral equation:

$$
u(t)= \begin{cases}u_{0}+\int_{0}^{t} \frac{(t-s)^{\alpha-1}}{\Gamma(\alpha)} f(s, u(s)) d s, & t \in\left[0, t_{1}\right), \\ u_{0}+I_{1}\left(u\left(t_{1}\right)\right)+\int_{0}^{t} \frac{(t-s)^{\alpha-1}}{\Gamma(\alpha)} f(s, u(s)) d s, & t \in\left[t_{1}, t_{2}\right), \\ u_{0}+I_{1}\left(u\left(t_{1}\right)\right)+I_{2}\left(u\left(t_{2}\right)\right)+\int_{0}^{t} \frac{(t-s)^{\alpha-1}}{\Gamma(\alpha)} f(s, u(s)) d s, & t \in\left[t_{2}, t_{3}\right), \\ \vdots & \\ u_{0}+\sum_{k=1}^{m} I_{k}\left(u\left(t_{k}\right)\right)+\int_{0}^{t} \frac{(t-s)^{\alpha-1}}{\Gamma(\alpha)} f(s, u(s)) d s, & t \in\left[t_{m}, T\right] .\end{cases}
$$

Finally, we agree with the second approach, which the concept of solutions for impulsive fractional differential equations has been given by (1.3). Furthermore, we point out an interesting recently published paper written by Liu and Ahmad [24], where the formula of solutions for semilinear impulsive fractional Cauchy problems (see (20) in [24]) is coincident with ours (see Lemma 3.1), if one imposes the requirement that the semilinear term and impulsive term have the same expression in a given finite interval. This paper very thoroughly and deeply explains the meaning of solutions for impulsive fractional differential equations from several points of view, and in this way it also supports our approach. Particularly, Shu and Shi [14] agree with the formula of the solution in [18-20, 25], where the formula of solutions for the semilinear impulsive fractional (see Theorem 4.2 in [14]) is coincident with the formula of the solution in [7-10,12, 13, 15-19, 21-23].

On the other hand, as far as we know there are few papers studied the fractional evolution equations with noncompact semigroup. Recently, Wang et al. [13] discussed the local existence of mild solutions for nonlocal problem of fractional evolution equations in the situation that $-A$ generates a noncompact analytic semigroup. Chen et al. [26] investigated the existence of saturated mild solutions for the initial value problem of fractional evolution equations in the situation that $-A$ generates an equicontinuous $C_{0}$-semigroup. However, periodic boundary value problem for impulsive fractional evolution equations have not been studied extensively. In [27], Yu et al. studied periodic boundary value problem for impulsive fractional evolution equations:

$$
\left\{\begin{array}{l}
{ }^{c} D_{0^{+}}^{\alpha} u(t)=A u(t)+f(t, u(t)), \quad \alpha \in(0,1), t \in[0, T], t \neq t_{k}, \\
u\left(t_{k}^{+}\right)=u\left(t_{k}^{-}\right)+y_{k}, \quad k=1,2, \ldots, m, \\
u(0)=u(T)
\end{array}\right.
$$

in Banach space $E, A: D(A) \subset E \rightarrow E$ is generator of a $C_{0}$-semigroup $T(t)(t \geq 0)$ in $E$ and $-A$ generates a compact $C_{0}$-semigroup. 
Shu et al. [28] investigated S-asymptotically $\omega$-positive periodic solutions to the following fractional partial neutral differential equations:

$$
\left\{\begin{array}{l}
{ }^{c} D_{t}^{\alpha}\left(u(t)+F\left(t, u_{t}\right)\right)+A(u(t))=G\left(t, u_{t}\right), \quad t \geq 0, \\
u(0)=\varphi \in \mathscr{B}
\end{array}\right.
$$

where ${ }^{c} D_{t}^{\alpha}$ is the Caputo fractional derivative with $0<\alpha<1$ and $-A$ is the infinitesimal generator of an analytic semigroup $\{T(t)\}_{t \geq 0}$.

Wang et al. $[15,16]$ discussed Ulam-Hyers stability for fractional equations and studied several types of such equations with various boundary value conditions as well, concept of solutions, existence results and examples are presented in [17].

However, in [29, 30], Mu et al. use the monotone iterative technique to investigate the existence and uniqueness of mild solutions of the impulsive fractional evolution equations in an order Banach space $E$ :

$$
\left\{\begin{array}{l}
{ }^{c} D_{t}^{\alpha} u(t)+A u(t)=f(t, u(t)), \quad t \in J, t \neq t_{k}, \\
\left.\Delta u\right|_{t=t_{k}}=I_{k}\left(u\left(t_{k}\right)\right), \quad k=1,2, \ldots, m, \\
u(0)=x_{0} \in E
\end{array}\right.
$$

and

$$
\left\{\begin{array}{l}
{ }^{c} D_{t}^{\alpha} u(t)+A u(t)=f(t, u(t)), \quad t \in J, t \neq t_{k} \\
\left.\Delta u\right|_{t=t_{k}}=I_{k}\left(u\left(t_{k}\right)\right), \quad k=1,2, \ldots, m \\
u(0)+g(u)=x_{0} \in E
\end{array}\right.
$$

where ${ }^{c} D_{t}^{\alpha}$ is the Caputo fractional derivative of order $\alpha \in(0,1), A: D(A) \subset E \rightarrow E$ be a closed linear operator and $-A$ generates a $C_{0}$-semigroup $T(t)(t \geq 0)$.

Unfortunately, these papers have some essential flaw (see Lemma 2.7 in [29] and Lemma 2.10 in [30] are not correct). Thus their main results are not correct too. Fortunately, in [7-10,12, 13, 15-19, 21-23], Wang et al. gave a new concept of solution for some impulsive differential equations with fractional derivative, which is a correction of that of piecewise continuous solutions used in $[4,6,11,29,30]$.

Furthermore, to the best of our knowledge, the theory of periodic boundary value problems for nonlinear impulsive fractional evolution equations is still in the initial stages and many aspects of this theory need to be explored; motivated by the above discussion, in this paper, we use some fixed point theorems and a measure of noncompactness to discuss the existence of mild solutions for the periodic boundary value problem (PBVP) of impulsive fractional evolution equations of Volterra type in an ordered Banach space $E$,

$$
\left\{\begin{array}{l}
{ }^{c} D_{0^{+}}^{\alpha} u(t)+A u(t)=f(t, u(t), F u(t), G u(t)), \quad t \in J, t \neq t_{k}, \\
\left.\Delta u\right|_{t=t_{k}}=I_{k}\left(u\left(t_{k}\right)\right), \quad k=1,2, \ldots, m, \\
u(0)=u(\omega),
\end{array}\right.
$$

where ${ }^{c} D_{0^{+}}^{\alpha}$ is the Caputo fractional derivative of order $\alpha \in(0,1)$ with the lower limit zero, $A: D(A) \subset E \rightarrow E$ be a closed linear operator and $-A$ generates a $C_{0}$-semigroup 
$T(t)(t \geq 0)$ in $E ; f \in C(J \times E \times E \times E, E), I_{k} \in C(E, E)$ is an impulsive function, $k=1,2, \ldots, m$; $J=[0, \omega], J^{\prime}=J \backslash\left\{t_{1}, t_{2}, \ldots, t_{m}\right\}, J_{0}=\left[0, t_{1}\right], J_{k}=\left(t_{k}, t_{k+1}\right]$, the $\left\{t_{k}\right\}$ satisfy $0=t_{0}<t_{1}<t_{2}<\cdots<$ $t_{m}<t_{m+1}=\omega, m \in N ; \Delta u\left(t_{k}\right)=u\left(t_{k}^{+}\right)-u\left(t_{k}^{-}\right), \Delta u^{\prime}\left(t_{k}\right)=u^{\prime}\left(t_{k}^{+}\right)-u^{\prime}\left(t_{k}^{-}\right), u\left(t_{k}^{+}\right)$and $u\left(t_{k}^{-}\right)$represent the right and left limits of $u(t)$ at $t=t_{k}$, respectively, and

$$
\begin{array}{ll}
F u(t)=\int_{0}^{t} K(t, s) u(s) d s, & K \in C\left(D_{1}, R^{+}\right), \\
G u(t)=\int_{0}^{\omega} H(t, s) u(s) d s, & H \in C\left(D_{0}, R^{+}\right),
\end{array}
$$

with $D_{1}=\left\{(t, s) \in R^{2}: 0 \leq s \leq t \leq \omega\right\}, D_{0}=\left\{(t, s) \in R^{2}: 0 \leq t, s \leq \omega\right\}, K_{0}=\max _{(t, s) \in D_{1}} K(t, s)$, $H_{0}=\max _{(t, s) \in D_{0}} H(t, s)$.

The rest of this paper is organized as follows: In Section 2 we recall some basic wellknown results and introduce some notations. In Section 3 we discuss the existence theorems of solutions for periodic boundary value problem (1.4). Two examples will be presented in Section 4 illustrating our results.

\section{Preliminaries}

In this section, we briefly recall some basic well=known results which will be used in the sequel.

Let $E$ be a Banach space with the norm $\|\cdot\|$ and $C(J, E)$ denote the Banach space of all continuous $E$-value functions on interval $J$ with the norm $\|u\|=\max _{t \in J}\|u(t)\|$.

Let $P C(J, E)=\left\{u: J \rightarrow E, u(t)\right.$ is continuous at $t \neq t_{k}$, and left continuous at $t=t_{k}$, and $u\left(t_{k}^{+}\right)$exists, $\left.k=1,2, \ldots, m\right\}$. Evidently, $P C(J, E)$ is a Banach space with the norm $\|u\|=$ $\sup _{t \in J}\|u(t)\|$. Set $C^{\alpha}(J, E)=\left\{\left.u \in C(J, E)\right|^{c} D_{0^{+}}^{\alpha} u\right.$ exists and $\left.{ }^{c} D_{0^{+}}^{\alpha} u \in C(J, E)\right\}$. Let $A: D(A) \subset$ $E \rightarrow E$ be a closed linear operator and $-A$ generate a $C_{0}$-semigroup $T(t)(t \geq 0)$ in $E$. Then there exist constants $N>0$ and $\delta \in \mathbb{R}$ such that

$$
\|T(t)\| \leq N e^{\delta t}, \quad t \geq 0
$$

and $\delta_{0}$ can also be expressed by $\delta_{0}=\lim _{t \rightarrow+\infty} \sup \frac{\ln \|T(t)\|}{t}$, then $\delta_{0}$ is called a growth index of the $C_{0}$-semigroup $T(t)(t \geq 0)$. If $\delta_{0}<0$, then $T(t)(t \geq 0)$ is called an exponentially stable $C_{0}$-semigroup.

Definition 2.1 A $C_{0}$-semigroup $T(t)(t \geq 0)$ is said to be exponentially stable in $E$ if there exist constants $N_{1} \geq 1$ and $\delta>0$ such that

$$
\|T(t)\| \leq N_{1} e^{-\delta t}, \quad t \geq 0
$$

For completeness we recall the definition of the Caputo derivative of fractional order.

Definition 2.2 The fractional integral of order $\alpha$ of a function $f:[0, \infty) \rightarrow R$ is defined as

$$
I_{0^{+}}^{\alpha}=\frac{1}{\Gamma(\alpha)} \int_{0}^{t}(t-s)^{\alpha-1} f(s) d s, \quad t>0, \alpha>0,
$$

provided the right side is point-wise defined on $(0, \infty)$, where $\Gamma(\cdot)$ is the gamma function. 
Definition 2.3 The Riemann-Liouville derivative of order $\alpha$ with the lower limit zero for a function $f:[0, \infty) \rightarrow R$ can be written as

$$
D_{0^{+}}^{\alpha} f(t)=\frac{1}{\Gamma(n-\alpha)} \frac{d^{n}}{d t^{n}} \int_{0}^{t} \frac{f(s)}{(t-s)^{\alpha+1-n}} d s, \quad t>0, n-1<\alpha<n .
$$

Definition 2.4 The Caputo fractional derivative of order $\alpha$ for a function $f:[0, \infty) \rightarrow R$ can be written as

$$
{ }^{c} D_{0^{+}}^{\alpha} f(t)=D_{0^{+}}^{\alpha}\left[f(t)-\sum_{k=0}^{n-1} \frac{t^{k}}{k !} f^{(k)}(0)\right], \quad t>0, n-1<\alpha<n,
$$

where $n=[\alpha]+1$ and $[\alpha]$ denotes the integer part of $\alpha$.

Remark 2.1 In the case $f(t) \in C^{n}[0, \infty)$, then

$$
{ }^{c} D_{0^{+}}^{\alpha} f(t)=\frac{1}{\Gamma(n-\alpha)} \int_{0}^{t}(t-s)^{n-\alpha-1} f^{(n)}(s) d s=I_{0+}^{n-\alpha} f^{n}(t), \quad t>0, n-1<\alpha<n .
$$

That is to say that Definition 2.4 is just the usual Caputo's fractional derivative. In this paper, we consider an impulsive problem, so Definition 2.4 is appropriate.

Remark 2.2 If $u$ is an abstract function with values in $E$, then the integrals which appear in Definitions 2.3 and 2.4 are taken in Bochner's sense.

In this paper we adopt the following definition of mild solutions, which comes from [12, $17,18,21,24]$.

Definition 2.5 By a mild solution of the initial value problem

$$
\left\{\begin{array}{l}
{ }^{c} D_{0^{+}}^{\alpha} u(t)+A u(t)=f(t, u(t), F u(t), G u(t)), \quad t \in J^{\prime} \\
\left.\Delta u\right|_{t=t_{k}}=I_{k}\left(u\left(t_{k}\right)\right), \quad k=1,2, \ldots, m, \\
u(0)=u_{0}
\end{array}\right.
$$

on $J$, we mean a function $u \in P C(J, E)$ satisfying

$$
\left\{\begin{array}{l}
\mathscr{T}_{\alpha}(t) u_{0}+\int_{0}^{t}(t-s)^{\alpha-1} \mathscr{S}_{\alpha}(t-s) f(s, u(s), F u(s), G u(s)) d s, \quad t \in\left[0, t_{1}\right], \\
\mathscr{T}_{\alpha}(t) u_{0}+\mathscr{T}_{\alpha}\left(t-t_{1}\right) I_{1}\left(u\left(t_{1}\right)\right) \\
\quad+\int_{0}^{t}(t-s)^{\alpha-1} \mathscr{S}_{\alpha}(t-s) f(s, u(s), F u(s), G u(s)) d s, \quad t \in\left(t_{1}, t_{2}\right], \\
\vdots \\
\quad \mathscr{T}_{\alpha}(t) u_{0}+\sum_{i=1}^{m} \mathscr{T}_{\alpha}\left(t-t_{i}\right) I_{i}\left(u\left(t_{i}\right)\right) \\
\quad+\int_{0}^{t}(t-s)^{\alpha-1} \mathscr{S}_{\alpha}(t-s) f(s, u(s), F u(s), G u(s)) d s, \quad t \in\left(t_{m}, \omega\right],
\end{array}\right.
$$


where

$$
\begin{aligned}
& \mathscr{T}_{\alpha}(t)=\int_{0}^{\infty} \theta_{\alpha}(s) T\left(t^{\alpha} s\right) d s, \quad \mathscr{S}_{\alpha}(t)=\alpha \int_{0}^{\infty} s \theta_{\alpha}(s) T\left(t^{\alpha} s\right) d s, \\
& \theta_{\alpha}(s)=\frac{1}{\pi \alpha} \sum_{n=1}^{\infty}(-s)^{n-1} \frac{\Gamma(n \alpha+1)}{n !} \sin (n \pi \alpha), \quad s \in(0, \infty),
\end{aligned}
$$

are the functions of Wright type defined on $(0, \infty)$ satisfying

$$
\theta_{\alpha}(s) \geq 0, \quad s \in(0, \infty), \quad \int_{0}^{\infty} \theta_{\alpha}(s) d s=1,
$$

and

$$
\int_{0}^{\infty} s^{v} \theta_{\alpha}(s) d s=\frac{\Gamma(1+v)}{\Gamma(1+\alpha v)}, \quad v \in[0,1] .
$$

We first give the following lemmas to be used in proving our main results, which can be found in [12].

Lemma 2.1 The operators $\mathscr{T}_{\alpha}(t)$ and $\mathscr{S}_{\alpha}(t)(t \geq 0)$ have the following properties:

(i) For any fixed $t \geq 0, \mathscr{T}_{\alpha}(t)$ and $\mathscr{S}_{\alpha}(t)$ are linear and bounded operators, i.e., for any $u \in E$,

$$
\left\|\mathscr{T}_{\alpha}(t) u\right\| \leq M\|u\|, \quad\left\|\mathscr{S}_{\alpha}(t) u\right\| \leq \frac{M}{\Gamma(\alpha)}\|u\| .
$$

(ii) For every $u \in E, t \rightarrow \mathscr{T}_{\alpha}(t) u$ and $t \rightarrow \mathscr{S}_{\alpha}(t) u$ are continuous functions from $[0, \infty)$ into $E$.

(iii) The operators $\mathscr{T}_{\alpha}(t)$ and $\mathscr{S}_{\alpha}(t)$ are strongly continuous for all $t \geq 0$.

(iv) If $T(t)(t \geq 0)$ is an equicontinuous semigroup, $\mathscr{T}_{\alpha}(t)$ and $\mathscr{S}_{\alpha}(t)$ are equicontinuous in $E$ for $t>0$.

(v) For every $t>0, \mathscr{T}_{\alpha}(t)$ and $\mathscr{S}_{\alpha}(t)$ are compact operators if $T(t)$ is compact.

Suppose that here the bounded operator $B: E \rightarrow E$ exists given by

$$
B=\left[I-\mathscr{T}_{\alpha}(\omega)\right]^{-1} .
$$

We present sufficient conditions for the existence and boundedness of the operator $B$.

Lemma 2.2 (see Theorem 3.3 and Remark 3.4 [23]) The operator B defined in (2.4) exists and is bounded, if one of the following three conditions holds:

(i) $T(t)$ is compact for each $t>0$ and the homogeneous linear nonlocal problem

$$
\left\{\begin{array}{l}
{ }^{c} D_{0^{+}}^{\alpha} u(t)=A u(t), \quad t \in J, \\
u(0)=u(\omega),
\end{array}\right.
$$

has no non-trivial mild solutions.

(ii) If $\left\|\mathscr{T}_{\alpha}(\omega)\right\|<1$, then the operator $I-\mathscr{T}_{\alpha}(\omega)$ is invertible and $\left[I-\mathscr{T}_{\alpha}(\omega)\right]^{-1} \in L_{b}(E)$. 
(iii) If $\|T(t)\|<1$ for $t \in(0, \omega]$, then $\mathscr{T}_{\alpha}(n \omega) \rightarrow 0$ as $n \rightarrow \infty$ and the operator $I-\mathscr{T}_{\alpha}(\omega)$ is invertible and $\left[I-\mathscr{T}_{\alpha}(\omega)\right]^{-1} \in L_{b}(E)$, where $L_{b}(E)$ denotes the space of bounded linear operators from $E$ to $E$.

Now, we recall some properties of the measure of noncompactness will be used later. Let $\alpha(\cdot)$ denote the Kuratowski measure of noncompactness of the bounded set. For any $B \subset C(J, E)$ and $t \in J$, set $B(t)=\{u(t): u \in B\} \subset E$. If $B$ is bounded in $C(J, E)$, then $B(t)$ is bounded in $E$, and $\alpha(B(t)) \leq \alpha(B)$.

Lemma 2.3 ([31]) Let $E$ be a Banach space, and let $B \subset E$ be bounded. Then there exists a countable set $B_{0} \subset B$, such that $\alpha(B) \leq 2 \alpha\left(B_{0}\right)$.

Lemma 2.4 ([32]) Let $E$ be a Banach space, and let $B \subset C(J, E)$ is equicontinuous and bounded, then $\alpha(B(t))$ is continuous on $J$, and $\alpha(B)=\max _{t \in J} \alpha(B(t))$.

Lemma 2.5 ([33]) Let $B=\left\{u_{n}\right\} \subset P C(J, E)$ be a bounded and countable set. Then $\alpha(B(t))$ is Lebesgue integral on $J$, and

$$
\alpha\left(\left\{\int_{J} u_{n}(t) d t: n \in \mathbb{N}\right\}\right) \leq 2 \int_{J} \alpha(B(t)) d t
$$

Lemma 2.6 (Sadovskii's fixed point theorem) Let $E$ be a Banach space and $\Omega_{0}$ be a nonempty bounded convex closed set in $E$. If $Q: \Omega_{0} \rightarrow \Omega_{0}$ is a condensing mapping, then $Q$ has a fixed point in $\Omega_{0}$.

\section{Main results}

In this section, we will present some main results. Before stating and proving these results, we introduce some notations and lemmas which are used in this sequel. For $B \subset C(J, E)$, let $B(t)=\{u(t): u \in B\}$ and denote $B_{r}(J)=\{u \in P C(J, E):\|u\| \leq r\}$.

Definition 3.1 An abstract function $u \in P C(J, E) \cap C^{\alpha}\left(J^{\prime}, E\right) \cap C\left(J^{\prime}, E_{1}\right)$ is called a solution of the PBVP (1.4) if $u(t)$ satisfies all the equalities of (1.4).

Lemma 3.1 Let $T(t)(t \geq 0)$ be an exponentially stable $C_{0}$-semigroup in $E$ generated by $-A$, then the PBVP (1.1) has a unique mild solution $u \in P C(J, E)$ given by

$$
u(t)= \begin{cases}\mathscr{T}_{\alpha}(t) R(u)+\int_{0}^{t}(t-s)^{\alpha-1} \mathscr{S}_{\alpha}(t-s) f(s, u(s), F u(s), G u(s)) d s, \\ & t \in\left[0, t_{1}\right], \\ & \mathscr{T}_{\alpha}(t) R(u)+\mathscr{T}_{\alpha}\left(t-t_{1}\right) I_{1}\left(u\left(t_{1}\right)\right) \\ & \quad+\int_{0}^{t}(t-s)^{\alpha-1} \mathscr{S}_{\alpha}(t-s) f(s, u(s), F u(s), G u(s)) d s, \quad t \in\left(t_{1}, t_{2}\right], \\ \vdots & \\ & \mathscr{T}_{\alpha}(t) R(u)+\sum_{i=1}^{m} \mathscr{T}_{\alpha}\left(t-t_{i}\right) I_{i}\left(u\left(t_{i}\right)\right) \\ & +\int_{0}^{t}(t-s)^{\alpha-1} \mathscr{S}_{\alpha}(t-s) f(s, u(s), F u(s), G u(s)) d s, \quad t \in\left(t_{m}, \omega\right],\end{cases}
$$


where

$$
R(u)=\left\{\begin{aligned}
B\left[\int_{0}^{\omega}(\omega-s)^{\alpha-1} \mathscr{S}_{\alpha}(\omega-s) f(s, u(s), F u(s), G u(s)) d s\right], \quad t \in\left[0, t_{1}\right] \\
B\left[\int_{0}^{\omega}(\omega-s)^{\alpha-1} \mathscr{S}_{\alpha}(\omega-s) f(s, u(s), F u(s), G u(s)) d s\right. \\
\left.\quad+\mathscr{T}_{\alpha}\left(\omega-t_{1}\right) I_{1}\left(u\left(t_{1}\right)\right)\right], \quad t \in\left(t_{1}, t_{2}\right], \\
B\left[\int_{0}^{\omega}(\omega-s)^{\alpha-1} \mathscr{S}_{\alpha}(\omega-s) f(s, u(s), F u(s), G u(s)) d s\right. \\
\left.\quad+\sum_{i=1}^{m} \mathscr{T}_{\alpha}\left(\omega-t_{i}\right) I_{i}\left(u\left(t_{i}\right)\right)\right], \quad t \in\left(t_{m}, \omega\right]
\end{aligned}\right.
$$

and $\mathscr{T}_{\alpha}, \mathscr{S}_{\alpha}$ are given by (2.3).

Proof For any $u \in P C(J, E)$, by Definition 2.5 and the proof of [12], we know easily that the initial value problem of the impulsive fractional evolution equation

$$
\left\{\begin{array}{l}
{ }^{c} D_{0^{+}}^{\alpha} u(t)+A u(t)=f(t, u(t), F u(t), G u(t)), \quad t \in J^{\prime} \\
\left.\Delta u\right|_{t=t_{k}}=I_{k}\left(u\left(t_{k}\right)\right), \quad k=1,2, \ldots, m, \\
u(0)=u_{0}
\end{array}\right.
$$

has a unique mild solution $u \in P C(J, E)$ given by

$$
u(t)=\left\{\begin{array}{l}
\mathscr{T}_{\alpha}(t) u_{0}+\int_{0}^{t}(t-s)^{\alpha-1} \mathscr{S}_{\alpha}(t-s) f(s, u(s), F u(s), G u(s)) d s, \quad t \in\left[0, t_{1}\right], \\
\mathscr{T}_{\alpha}(t) u_{0}+\mathscr{T}_{\alpha}\left(t-t_{1}\right) I_{1}\left(u\left(t_{1}\right)\right) \\
\quad+\int_{0}^{t}(t-s)^{\alpha-1} \mathscr{S}_{\alpha}(t-s) f(s, u(s), F u(s), G u(s)) d s, \quad t \in\left(t_{1}, t_{2}\right], \\
\vdots \\
\quad \mathscr{T}_{\alpha}(t) u_{0}+\sum_{i=1}^{m} \mathscr{T}_{\alpha}\left(t-t_{i}\right) I_{i}\left(u\left(t_{i}\right)\right) \\
\quad+\int_{0}^{t}(t-s)^{\alpha-1} \mathscr{S}_{\alpha}(t-s) f(s, u(s), F u(s), G u(s)) d s, \quad t \in\left(t_{m}, \omega\right],
\end{array}\right.
$$

where $\mathscr{T}_{\alpha}$ and $\mathscr{S}_{\alpha}$ are given by (2.3).

We show that the PBVP (1.4) has a unique mild solution $u \in P C(J, E)$ given by (3.1). If a function $u \in P C(J, E)$ defined by (2.2) is a solution of the PBVP (1.4) and $u_{0}=u(\omega)$, then

$$
\begin{aligned}
& {\left[I-\mathscr{T}_{\alpha}(\omega)\right] u_{0}} \\
& \quad=\int_{0}^{\omega}(\omega-s)^{\alpha-1} \mathscr{S}_{\alpha}(\omega-s) f(s, u(s), F u(s), G u(s)) d s \\
& \quad+\sum_{i=1}^{m} \mathscr{T}_{\alpha}\left(\omega-t_{i}\right) I_{i}\left(u\left(t_{i}\right)\right), \quad t \in J_{k}, k=0,1,2, \ldots, m .
\end{aligned}
$$

Since $T(t)(t \geq 0)$ is exponentially stable, we define an equivalent norm in $E$ by

$$
|x|=\sup _{t \geq 0}\left\|e^{\delta t} T(t) x\right\|
$$

Then $\|x\| \leq|x| \leq N\|x\|$ and $|T(t)|<e^{-\delta t}(t \geq 0)$, and especially, $T(\omega)<e^{-\delta \omega}<1$. It follows that $I-T(\omega)$ has a bounded inverse operator $[I-T(\omega)]^{-1}$ by virtue of Lemma 2.2(iii). 
Hence we choose

$$
\begin{aligned}
u_{0}= & B\left[\int_{0}^{\omega}(\omega-s)^{\alpha-1} \mathscr{S}_{\alpha}(\omega-s) f(s, u(s), F u(s), G u(s)) d s\right. \\
& \left.+\sum_{i=1}^{m} \mathscr{T}_{\alpha}\left(\omega-t_{i}\right) I_{i}\left(u\left(t_{i}\right)\right)\right] \triangleq R(u) .
\end{aligned}
$$

Then $u_{0}$ is the unique initial value of the problem (2.1) in $E$, which satisfies $u(0)=u_{0}=$ $u(\omega)$. It follows that the mild solution $u$ of the problem (2.1) corresponding to initial value $u(0)=u_{0}=R(h)$ is just the mild solution of the PBVP (1.4). Therefore, the conclusion of Lemma 3.1 holds.

To prove our main results, the following lemma is needed.

Lemma 3.2 Assume that $\alpha>0, m \in C\left(J, R^{+}\right)$satisfies

$$
\begin{aligned}
m(t) \leq & M_{1} \int_{0}^{t}(t-s)^{\alpha-1} m(s) d s+M_{2} \int_{0}^{t}(t-s)^{\alpha-1} m(s) d s \\
& +M_{3} \int_{0}^{\omega}(t-s)^{\alpha-1} m(s) d s, \quad t \in J
\end{aligned}
$$

where $M_{i} \geq 0(i=1,2,3)$ are constants. Then $m(t) \equiv 0$ for $t \in J$ provided the following condition holds: (i) $\frac{\left(M_{1}+M_{2}+M_{3}\right) \omega^{\alpha}}{\alpha}<1$.

Proof Let us suppose that (i) holds. Then, from (3.3),

$$
m(t) \leq\left(M_{1}+M_{2}+M_{3}\right) \int_{0}^{\omega}(t-s)^{\alpha-1} m(s) d s, \quad t \in J .
$$

If follows by integrating the above inequality that

$$
\int_{0}^{\omega} m(s) d s \leq \frac{\left(M_{1}+M_{2}+M_{3}\right) \omega^{\alpha}}{\alpha} \int_{0}^{\omega} m(s) d s
$$

and by assumption (i), implies

$$
\int_{0}^{\omega} m(s) d s=0
$$

and so $m(t) \equiv 0, t \in J$. The proof of this lemma is complete.

Throughout this paper, we introduce the following hypotheses:

(H1) $f: J \times E \times E \times E \rightarrow E$ is a continuous function and there exist a Lebesgue-integrable function $M_{r}: J \rightarrow R^{+}$and nondecreasing continuous function $\Omega:[0, \infty) \rightarrow(0, \infty)$ such that

$$
\|f(t, x, y, z)\| \leq M_{r}(t) \Omega(r)
$$

for all $t \in J,(x, y, z) \in B_{r} \times B_{r} \times B_{r}$. 
(H2) The functions $I_{k}: E \rightarrow E$ are continuous and there exists a constant $\rho>0$ such that

$$
\left\|I_{k}(u)\right\| \leq \rho\|u\| \quad \text { for all } u, v \in B_{r} \text { and } k=1,2, \ldots, m \text {. }
$$

(H3) There exists a positive solution $r_{0}$ of the inequality

$$
M\left(M M^{*}+1\right)\left(\frac{\left\|M_{r_{0}}\right\| \Omega\left(r_{0}\right) \omega^{\alpha}}{\Gamma(\alpha+1)}+m \rho\right) \leq r_{0},
$$

where $M^{*}=\|B\|$.

(H4) There exists a constant $0<L_{1}<\frac{\Gamma(\alpha+1)\left[1-2 M\left(M M^{*}+1\right) \sum_{k=1}^{m} M_{k}\right]}{4 M^{2}\left(M M^{*}+1\right)\left(1+\omega K_{0}+\omega H_{0}\right) \omega^{\alpha}}$ such that, for any bounded and equicontinuous sets $V_{i} \subset C(J, E)(i=1,2,3)$ and $t \in J$

$$
\beta\left(f\left(t, V_{1}, V_{2}, V_{3}\right)\right) \leq L_{1} \sum_{i=1}^{3} \beta\left(V_{i}\right) .
$$

(H5) There exists $M_{k}>0, k=1,2, \ldots, m$ with $\sum_{k=1}^{m} M_{k}<\frac{1}{2 M\left(M M^{*}+1\right)}$ such that

$$
\alpha\left(\left\{I_{k}\left(x_{n}\left(t_{k}\right)\right)\right\}\right) \leq M_{k} \alpha\left(x_{n}\left(t_{k}\right)\right),
$$

for any countable subsets $\left\{x_{n}\right\} \subset P C(J, E)$.

Theorem 3.1 Let E be a Banach space, $A: D(A) \subset E \rightarrow E$ be a closed linear operator and $-A$ generate an equicontinuous $C_{0}$-semigroup $T(t)(t \geq 0)$ in $E$. Assume that the conditions (H1)-(H5) are satisfied. Then the PBVP (1.4) has at least one mild solution on J.

Proof Choosing

$$
r_{0} \geq M\left(M M^{*}+1\right)\left(\frac{\left\|M_{r_{0}}\right\| \Omega(R) \omega^{\alpha}}{\Gamma(\alpha+1)}+m \rho\right)
$$

Consider $B_{r_{0}}(J)=\left\{u \in P C(J, E):\|u(t)\| \leq r_{0}, t \in J\right\}$, then $B_{r_{0}}(J)$ is a closed, bounded and convex subset of $P C(J, E)$.

From Lemma 3.1, we define the mapping $Q: P C(J, E) \rightarrow P C(J, E)$ by

$$
Q u(t)= \begin{cases}\mathscr{T}_{\alpha}(t) R(u)+\int_{0}^{t}(t-s)^{\alpha-1} \mathscr{S}_{\alpha}(t-s) f(s, u(s), F u(s), G u(s)) d s, \\ & t \in\left[0, t_{1}\right], \\ & \mathscr{T}_{\alpha}(t) R(u)+\mathscr{T}_{\alpha}\left(t-t_{1}\right) I_{1}\left(u\left(t_{1}\right)\right) \\ & \quad+\int_{0}^{t}(t-s)^{\alpha-1} \mathscr{S}_{\alpha}(t-s) f(s, u(s), F u(s), G u(s)) d s, \quad t \in\left(t_{1}, t_{2}\right], \\ \vdots & \\ & \mathscr{T}_{\alpha}(t) R(u)+\sum_{i=1}^{m} \mathscr{T}_{\alpha}\left(t-t_{i}\right) I_{i}\left(u\left(t_{i}\right)\right) \\ & \quad+\int_{0}^{t}(t-s)^{\alpha-1} \mathscr{S}_{\alpha}(t-s) f(s, u(s), F u(s), G u(s)) d s, \quad t \in\left(t_{m}, \omega\right],\end{cases}
$$

where $R(u)$ is given by (3.2). Obviously, $u$ is a mild solution of the PBVP (1.4) if and only if $u$ is a solution of the operator equation $u=Q u$. We will use Sadovskii's fixed point theorem 
to prove that the operator equation $u=Q u$ has a solution on $B_{r_{0}}(J)$. Our proof will be divided into four steps.

Step 1. We show that $Q B_{r_{0}}(J) \subset B_{r_{0}}(J)$.

Let $0<r_{1}<\min \left(r_{0}, \frac{r_{0}}{K_{0} \omega}\right)$, for any $u \in B_{r_{1}}(J)$ and $t \in J$, we have

$$
\|(F u)(s)\|=\left\|\int_{0}^{t} K(t, s) u(s) d s\right\| \leq K_{0} \int_{0}^{\omega}\|u(s)\| d s \leq K_{0} \omega r_{1} \leq r_{0} .
$$

So $F u \in B_{r_{0}}(J)$. Similarly, we prove $G u \in B_{r_{0}}(J)$.

For each $t \in J_{0}=\left[0, t_{1}\right]$, by Lemma 2.1(i) and (3.5), we have

$$
\begin{aligned}
\|(Q u)(t)\| & \leq\left\|\mathscr{T}_{\alpha}(t) R(u)+\int_{0}^{t}(t-s)^{\alpha-1} \mathscr{S}_{\alpha}(t-s) f(s, u(s), F u(s), G u(s)) d s\right\| \\
& \leq M\|R(u)\|+\frac{M \Omega\left(r_{0}\right)}{\Gamma(\alpha)} \int_{0}^{t}(t-s)^{\alpha-1} M_{r_{0}}(s) d s \\
& \leq M M^{*} \frac{M\left\|M_{r_{0}}\right\| \Omega\left(r_{0}\right) \omega^{\alpha}}{\Gamma(\alpha+1)}+\frac{M\left\|M_{r_{0}}\right\| \Omega\left(r_{0}\right) \omega^{\alpha}}{\Gamma(\alpha+1)} \\
& =M\left(M M^{*}+1\right) \frac{\left\|M_{r_{0}}\right\| \Omega\left(r_{0}\right) \omega^{\alpha}}{\Gamma(\alpha+1)} \\
& \leq r_{0} .
\end{aligned}
$$

By the same method, for each $t \in J_{k}=\left(t_{k}, t_{k+1}\right], k=1,2, \ldots, m$, we have

$$
\begin{aligned}
\|(Q u)(t)\| \leq & \mathscr{T}_{\alpha}(t) R(u)+\sum_{i=1}^{m} \mathscr{T}_{\alpha}\left(t-t_{i}\right) I_{i}\left(u\left(t_{i}\right)\right) \\
& +\int_{0}^{t}(t-s)^{\alpha-1} \mathscr{S}_{\alpha}(t-s) f(s, u(s), F u(s), G u(s)) d s \| \\
\leq & M\|R(u)\|+M \sup _{u \in B_{r_{0}}(J)} \sum_{i=1}^{m}\left\|I_{i}\left(u\left(t_{i}\right)\right)\right\|+\frac{M \Omega\left(r_{0}\right)}{\Gamma(\alpha)} \int_{0}^{t}(t-s)^{\alpha-1} M_{r_{0}}(s) d s \\
\leq & M M^{*}\left(\frac{M\left\|M_{r_{0}}\right\| \Omega\left(r_{0}\right) \omega^{\alpha}}{\Gamma(\alpha+1)}+M m \rho\right) \\
& +M \sup _{u \in B_{r_{0}}(J)} \sum_{i=1}^{m}\left\|I_{i}\left(u\left(t_{i}\right)\right)\right\|+\frac{M\left\|M_{r_{0}}\right\| \Omega\left(r_{0}\right) \omega^{\alpha}}{\Gamma(\alpha+1)} \\
\leq & M M^{*}\left(\frac{M\left\|M_{r_{0}}\right\| \Omega\left(r_{0}\right) \omega^{\alpha}}{\Gamma(\alpha+1)}+M m \rho\right)+\frac{M\left\|M_{r_{0}}\right\| \Omega\left(r_{0}\right) \omega^{\alpha}}{\Gamma(\alpha+1)}+M m \rho \\
= & M\left(M M^{*}+1\right)\left(\frac{\left\|M_{r_{0}}\right\| \Omega\left(r_{0}\right) \omega^{\alpha}}{\Gamma(\alpha+1)}+m \rho\right) \\
\leq & r_{0} .
\end{aligned}
$$

Hence, $\|Q u\| \leq r_{0}$, for any $u \in B_{r_{0}}(J)$, i.e., $Q u \in B_{r_{0}}(J)$.

Step 2. Now we show that $Q$ is continuous from $B_{r_{0}}(J)$ into $B_{r_{0}}(J)$. To show this, for any $u_{n}, u \in B_{r_{0}}(J), n=1,2, \ldots$, with $\lim _{n \rightarrow \infty}\left\|u_{n}-u\right\|=0$, we get

$$
\lim _{n \rightarrow \infty} u_{n}(t)=u(t),
$$


for all $t \in J$. Hence, by (H1), (H2), we have

$$
\begin{aligned}
& \lim _{n \rightarrow \infty} f\left(t, u_{n}(t),\left(F u_{n}\right)(t),\left(G u_{n}\right)(t)\right)=f(t, u(t),(F u)(t),(G u)(t)), \quad t \in J, \\
& \lim _{n \rightarrow \infty} I_{k}\left(u_{n}\left(t_{k}\right)\right)=I_{k}\left(u\left(t_{k}\right)\right), \quad k=1,2, \ldots, m .
\end{aligned}
$$

On the one hand, using (H1), we get, for each $t \in J$,

$$
\begin{aligned}
& (t-s)^{\alpha-1}\left\|f\left(s, u_{n}(s),\left(F u_{n}\right)(s),\left(G u_{n}\right)(s)\right)-f(s, u(s),(F u)(s),(G u)(s))\right\| \\
& \quad \leq(t-s)^{\alpha-1} 2 \Omega\left(r_{0}\right) M_{r_{0}}(s), \quad \text { a.e. in }[0, t) .
\end{aligned}
$$

On the other hand, the function $s \rightarrow(t-s)^{\alpha-1} 2 M_{R}(s)$ is integrable for $s \in[0, t)$ and $t \in J$. By Lebesgue dominated convergence theorem, we have

$$
\begin{aligned}
& \int_{0}^{t}(t-s)^{\alpha-1}\left\|f\left(s, u_{n}(s),\left(F u_{n}\right)(s),\left(G u_{n}\right)(s)\right)-f(s, u(s),(F u)(s),(G u)(s))\right\| d s \rightarrow 0, \\
& \quad \text { as } n \rightarrow \infty .
\end{aligned}
$$

For $t \in J_{0}=\left[0, t_{1}\right]$, we have

$$
\begin{aligned}
& \left\|\left(R u_{n}\right)(t)-(R u)(t)\right\| \\
& \quad \leq \frac{M M^{*} \omega^{\alpha}}{\Gamma(\alpha+1)}\left\|f\left(t, u_{n}(t),\left(F u_{n}\right)(t),\left(G u_{n}\right)(t)\right)-f(t, u(t),(F u)(t),(G u)(t))\right\| \\
& \quad \rightarrow 0, \quad \text { as } n \rightarrow \infty
\end{aligned}
$$

Then we have

$$
\begin{aligned}
&\left\|\left(Q u_{n}\right)(t)-(Q u)(t)\right\| \\
& \leq\left\|\mathscr{T}_{\alpha}(t)\right\| \cdot\left\|\left(R\left(u_{n}\right)(t)-R(u)(t)\right)\right\| \\
&+\int_{0}^{t}(t-s)^{\alpha-1}\left\|\mathscr{S}_{\alpha}(t-s)\right\| \\
& \quad \times\left\|f\left(s, u_{n}(s),\left(F u_{n}\right)(s),\left(G u_{n}\right)(s)\right)-f(s, u(s),(F u)(s),(G u)(s))\right\| d s \\
& \leq M\left\|\left(R\left(u_{n}\right)(t)-R(u)(t)\right)\right\| \\
& \quad+\frac{M}{\Gamma(\alpha)} \int_{0}^{t}(t-s)^{\alpha-1}\left\|f\left(s, u_{n}(s),\left(F u_{n}\right)(s),\left(G u_{n}\right)(s)\right)-f(s, u(s),(F u)(s),(G u)(s))\right\| d s \\
& \rightarrow 0, \quad \text { as } n \rightarrow \infty
\end{aligned}
$$

For $t \in J_{k}=\left(t_{k}, t_{k+1}\right]$, we have

$$
\begin{aligned}
& \left\|\left(R u_{n}\right)(t)-(R u)(t)\right\| \\
& \leq \frac{M M^{*} \omega^{\alpha}}{\Gamma(\alpha+1)}\left\|f\left(t, u_{n}(t),\left(F u_{n}\right)(t),\left(G u_{n}\right)(t)\right)-f(t, u(t),(F u)(t),(G u)(t))\right\| \\
& \quad+m M\left\|I_{k}\left(u_{n}\left(t_{k}\right)\right)-I_{k}\left(u\left(t_{k}\right)\right)\right\| \rightarrow 0, \quad \text { as } n \rightarrow \infty .
\end{aligned}
$$


Then we have

$$
\begin{aligned}
&\left\|\left(Q u_{n}\right)(t)-(Q u)(t)\right\| \\
& \leq\left\|\mathscr{T}_{\alpha}(t)\right\| \cdot\left\|\left(R\left(u_{n}\right)(t)-R(u)(t)\right)\right\| \\
&+\int_{0}^{t}(t-s)^{\alpha-1}\left\|\mathscr{S}_{\alpha}(t-s)\right\| \\
& \times\left\|f\left(s, u_{n}(s),\left(F u_{n}\right)(s),\left(G u_{n}\right)(s)\right)-f(s, u(s),(F u)(s),(G u)(s))\right\| d s \\
&+\sum_{k=1}^{m}\left\|\mathscr{T}_{\alpha}\left(t-t_{k}\right)\right\| \cdot\left\|I_{k}\left(u_{n}\left(t_{k}^{-}\right)\right)-I_{k}\left(u\left(t_{k}^{-}\right)\right)\right\| \\
& \leq M\left\|\left(R\left(u_{n}\right)(t)-R(u)(t)\right)\right\|+m M\left\|I_{k}\left(u_{n}\left(t_{k}^{-}\right)\right)-I_{k}\left(u\left(t_{k}^{-}\right)\right)\right\| \\
& \quad \frac{M}{\Gamma(\alpha)} \int_{0}^{t}(t-s)^{\alpha-1} \| f\left(s, u_{n}(s),\left(F u_{n}\right)(s),\left(G u_{n}\right)(s)\right) \\
&-f(s, u(s),(F u)(s),(G u)(s)) \| d s \\
& \rightarrow 0, \quad \text { as } n \rightarrow \infty,
\end{aligned}
$$

which implies that $Q u_{n} \rightarrow Q u$ uniformly on $J$ as $n \rightarrow \infty$ and so $Q: B_{r_{0}}(J) \rightarrow B_{r_{0}}(J)$ is a continuous operator.

Step 3. Now, we demonstrate that $\left\{Q u: u \in B_{r_{0}}(J)\right\}$ is equicontinuous. For any $u \in B_{r_{0}}(J)$ and $0 \leq t_{1}<t_{2} \leq \omega$, we get

$$
\begin{aligned}
\left\|(Q u)\left(t_{2}\right)-(Q u)\left(t_{1}\right)\right\| \\
=\left(\mathscr{T}_{\alpha}\left(t_{2}\right)-\mathscr{T}_{\alpha}\left(t_{1}\right)\right) R(u) \\
\quad+\int_{t_{1}}^{t_{2}}\left(t_{2}-s\right)^{\alpha-1} \mathscr{S}_{\alpha}\left(t_{2}-s\right) f(s, u(s),(F u)(s),(G u)(s)) d s \\
\quad+\int_{0}^{t_{1}}\left(\left(t_{2}-s\right)^{\alpha-1}-\left(t_{1}-s\right)^{\alpha-1}\right) \mathscr{S}_{\alpha}\left(t_{2}-s\right) f(s, u(s),(F u)(s),(G u)(s)) d s \\
\quad+\int_{0}^{t_{1}}\left(t_{1}-s\right)^{\alpha-1}\left(\mathscr{S}_{\alpha}\left(t_{2}-s\right)-\mathscr{S}_{\alpha}\left(t_{1}-s\right)\right) f(s, u(s),(F u)(s),(G u)(s)) d s \\
\quad+\sum_{k=1}^{m}\left(\mathscr{T}_{\alpha}\left(t_{2}-t_{k}\right)-\mathscr{T}_{\alpha}\left(t_{1}-t_{k}\right)\right) \times I_{k}\left(u\left(t_{k}^{-}\right)\right) \\
=I_{1}+I_{2}+I_{3}+I_{4}+I_{5} .
\end{aligned}
$$

Here we calculate

$$
\left\|(Q u)\left(t_{2}\right)-(Q u)\left(t_{1}\right)\right\| \leq \sum_{i=1}^{5}\left\|I_{i}\right\| .
$$

Therefore, we inspect that $\left\|I_{i}\right\|$ tend to 0 , when $t_{2}-t_{1} \rightarrow 0, i=1,2, \ldots, 5$.

For $I_{1}, I_{5}$, by Lemma 2.1(iv), we have

$$
\left\|I_{1}\right\| \rightarrow 0 \quad \text { as } t_{2}-t_{1} \rightarrow 0
$$




$$
\begin{aligned}
\left\|I_{5}\right\| & =\sum_{k=1}^{m}\left(\mathscr{T}_{\alpha}\left(t_{2}-t_{k}\right)-\mathscr{T}_{\alpha}\left(t_{1}-t_{k}\right)\right) \times I_{k}\left(u\left(t_{k}^{-}\right)\right) \\
& \leq m \rho\left\|\mathscr{T}_{\alpha}\left(t_{2}-t_{k}\right)-\mathscr{T}_{\alpha}\left(t_{1}-t_{k}\right)\right\| \\
& \rightarrow 0, \quad t_{2}-t_{1} \rightarrow 0 .
\end{aligned}
$$

For $I_{2}, I_{3}$ by Lemma 2.1(i) and (H1), we have

$$
\begin{aligned}
\left\|I_{2}\right\| & \leq \frac{M\left\|M_{r_{0}}\right\| \Omega\left(r_{0}\right)}{\Gamma(\alpha)} \int_{t_{1}}^{t_{2}}\left(t_{2}-s\right)^{\alpha-1} d s \leq \frac{M\left\|M_{r_{0}}\right\| \Omega\left(r_{0}\right)}{\Gamma(\alpha+1)}\left(t_{2}-t_{1}\right)^{\alpha} \rightarrow 0, \quad t_{2}-t_{1} \rightarrow 0 . \\
\left\|I_{3}\right\| & =\int_{0}^{t_{1}}\left(\left(t_{2}-s\right)^{\alpha-1}-\left(t_{1}-s\right)^{\alpha-1}\right) \mathscr{S}_{\alpha}\left(t_{2}-s\right) f(s, u(s),(F u)(s),(G u)(s)) d s \\
& \leq \frac{M\left\|M_{r_{0}}\right\| \Omega\left(r_{0}\right)}{\Gamma(\alpha)} \int_{0}^{t_{1}}\left(\left(t_{2}-s\right)^{\alpha-1}-\left(t_{1}-s\right)^{\alpha-1}\right) d s \\
& \leq \frac{M\left\|M_{r_{0}}\right\| \Omega\left(r_{0}\right)}{\Gamma(\alpha+1)}\left(t_{2}-t_{1}\right)^{\alpha} \\
& \rightarrow 0, \quad t_{2}-t_{1} \rightarrow 0 .
\end{aligned}
$$

For $I_{4}$, by Lemma 2.1(iv) and (H1), we have

$$
\begin{aligned}
\left\|I_{4}\right\| & =\int_{0}^{t_{1}}\left(t_{1}-s\right)^{\alpha-1}\left(\mathscr{S}_{\alpha}\left(t_{2}-s\right)-\mathscr{S}_{\alpha}\left(t_{1}-s\right)\right) f(s, u(s),(F u)(s),(G u)(s)) d s \\
& \leq\left\|M_{r_{0}}\right\| \Omega\left(r_{0}\right) \int_{0}^{t_{1}}\left(t_{1}-s\right)^{\alpha-1}\left\|\mathscr{S}_{\alpha}\left(t_{2}-s\right)-\mathscr{S}_{\alpha}\left(t_{1}-s\right)\right\| d s \\
& \rightarrow 0, \quad t_{2}-t_{1} \rightarrow 0 .
\end{aligned}
$$

In conclusion, $\left\|(Q u)\left(t_{2}\right)-(Q u)\left(t_{1}\right)\right\| \rightarrow 0$ as $t_{2}-t_{1} \rightarrow 0$, which implies that $Q\left(B_{r_{0}}(J)\right)$ is equicontinuous.

Let $B=\overline{c o} Q\left(B_{r_{0}}(J)\right)$. Then it is easy to verify that $Q$ maps $B$ into itself and $B \subset P C(J, E)$ is equicontinuous.

Step 4. Now, we prove that $Q: B \rightarrow B$ is a condensing operator. For any $W \subset B$, by Lemma 2.3, there exists a countable set $W_{0}=\left\{u_{n}\right\} \subset W$, such that

$$
\beta(Q(W)) \leq 2 \beta\left(Q\left(W_{0}\right)\right)
$$

By the equicontinuity of $B$, we know that $W_{0} \subset B$ is also equicontinuous.

By this fact that

$$
\int_{0}^{\omega} u(s) d s \in \omega \overline{c o}\{u(s) \mid s \in J\}, \quad u \in C(J, E),
$$

we have

$$
\begin{aligned}
& \beta\left(\left\{\int_{0}^{t} K(t, s) u(s) d s \mid u \in B, t \in J\right\}\right) \leq \omega K_{0} \beta(\{u(t) \mid u \in B, t \in J\}), \\
& \beta\left(\left\{\int_{0}^{\omega} H(t, s) u(s) d s \mid u \in B, t \in J\right\}\right) \leq \omega H_{0} \beta(\{u(t) \mid u \in B, t \in J\}) .
\end{aligned}
$$


For $t \in J_{0}=\left[0, t_{1}\right]$, by the definition of $Q,(3.8),(3.9)$ and Lemma 2.5, we have

$$
\begin{aligned}
\alpha\left(Q\left(W_{0}(t)\right)\right) & \alpha\left(\left\{\mathscr{T}_{\alpha}(t) R\left(u_{n}\right)+\int_{0}^{t}(t-s)^{\alpha-1} \mathscr{S}_{\alpha}(t-s)\left(f\left(s, u_{n}(s), F u_{n}(s), G u_{n}(s)\right)\right) d s: n \in N\right\}\right) \\
\leq & M \alpha\left(\left\{R\left(u_{n}\right): n \in N\right\}\right) \\
& +\frac{2 M}{\Gamma(\alpha)} \int_{0}^{t}(t-s)^{\alpha-1} \alpha\left(\left\{f\left(s, u_{n}(s), F u_{n}(s), G u_{n}(s)\right): n \in N\right\}\right) d s \\
\leq & M M^{*}\left[\frac{M L_{1}\left(1+\omega K_{0}+\omega H_{0}\right) \omega^{\alpha}}{\Gamma(\alpha+1)}\right] \alpha(W) \\
& +\frac{2 M}{\Gamma(\alpha)} \int_{0}^{t}(t-s)^{\alpha-1}\left(L_{1}\left[\alpha\left(W_{0}(s)\right)+\alpha\left(F\left(W_{0}\right)(s)\right)+\alpha\left(G\left(W_{0}\right)(s)\right)\right]\right) d s \\
\leq & M M^{*}\left[\frac{M L_{1}\left(1+\omega K_{0}+\omega H_{0}\right) \omega^{\alpha}}{\Gamma(\alpha+1)}\right] \alpha(W)+\frac{2 M L_{1}}{\Gamma(\alpha)} \int_{0}^{t}(t-s)^{\alpha-1} \alpha\left(W_{0}(s)\right) d s \\
& +\frac{2 M}{\Gamma(\alpha)} \omega L_{1} K_{0} \int_{0}^{t}(t-s)^{\alpha-1} \alpha\left(W_{0}(s)\right) d s+\frac{2 M}{\Gamma(\alpha)} \omega L_{1} H_{0} \int_{0}^{\omega}(t-s)^{\alpha-1} \alpha\left(W_{0}(s)\right) d s \\
< & M M^{*}\left[\frac{2 M L_{1}\left(1+\omega K_{0}+\omega H_{0}\right) \omega^{\alpha}}{\Gamma(\alpha+1)}\right] \alpha(W)+\left[\frac{2 M L_{1}\left(1+\omega K_{0}+\omega H_{0}\right) \omega^{\alpha}}{\Gamma(\alpha+1)}\right] \alpha(W) \\
= & \left(M M^{*}+1\right)\left[\frac{2 M L_{1}\left(1+\omega K_{0}+\omega H_{0}\right) \omega^{\alpha}}{\Gamma(\alpha+1)}\right] \alpha(W) .
\end{aligned}
$$

For $t \in J_{k}=\left(t_{k}, t_{k+1}\right], k=1,2, \ldots, m$, by the definition of $Q$, (3.8), (3.9) and Lemma 2.5, we have

$$
\begin{aligned}
\alpha\left(Q\left(W_{0}(t)\right)\right) & \\
= & \alpha\left(\left\{\mathscr{T}_{\alpha}(t) R\left(u_{n}\right)+\int_{0}^{t}(t-s)^{\alpha-1} \mathscr{S}_{\alpha}(t-s)\left(f\left(s, u_{n}(s), F u_{n}(s), G u_{n}(s)\right) d s\right.\right.\right. \\
& \left.\left.\left.+\sum_{k=1}^{m} \mathscr{T}_{\alpha}\left(t-t_{k}\right) I_{k}\left(u_{n}\left(t_{k}\right)\right): n \in N\right)\right\}\right) \\
\leq & M \alpha\left(\left\{R\left(u_{n}\right): n \in N\right\}\right) \\
& +\frac{2 M}{\Gamma(\alpha)} \int_{0}^{t}(t-s)^{\alpha-1} \alpha\left(\left\{f\left(s, u_{n}(s), F u_{n}(s), G u_{n}(s)\right): n \in N\right\}\right) d s \\
& +M \sum_{k=1}^{m} M_{k} \alpha\left(I_{k}\left(u_{n}\left(t_{k}\right)\right)\right) \\
\leq & M M^{*}\left[\frac{M L_{1}\left(1+\omega K_{0}+\omega H_{0}\right) \omega^{\alpha}}{\Gamma(\alpha+1)}+M \sum_{k=1}^{m} M_{k}\right] \alpha(W) \\
& +\frac{2 M}{\Gamma(\alpha)} \int_{0}^{t}(t-s)^{\alpha-1}\left(L _ { 1 } \left[\alpha\left(W_{0}(s)\right)+\alpha\left(F\left(W_{0}\right)(s)\right)\right.\right. \\
& \left.\left.+\alpha\left(G\left(W_{0}\right)(s)\right)\right]\right) d s+M \sum_{k=1}^{m} M_{k} \alpha\left(W_{0}\left(t_{k}\right)\right)
\end{aligned}
$$




$$
\begin{aligned}
\leq & M M^{*}\left[\frac{M L_{1}\left(1+\omega K_{0}+\omega H_{0}\right) \omega^{\alpha}}{\Gamma(\alpha+1)}+M \sum_{k=1}^{m} M_{k}\right] \alpha(W) \\
& +\frac{2 M L_{1}}{\Gamma(\alpha)} \int_{0}^{t}(t-s)^{\alpha-1} \alpha\left(W_{0}(s)\right) d s \\
& +\frac{2 M}{\Gamma(\alpha)} \omega L_{1} K_{0} \int_{0}^{t}(t-s)^{\alpha-1} \alpha\left(W_{0}(s)\right) d s+\frac{2 M}{\Gamma(\alpha)} \omega L_{1} H_{0} \int_{0}^{\omega}(t-s)^{\alpha-1} \alpha\left(W_{0}(s)\right) d s \\
& +M \sum_{k=1}^{m} M_{k} \alpha\left(W_{0}\left(t_{k}\right)\right) \\
\leq & M M^{*}\left[\frac{M L_{1}\left(1+\omega K_{0}+\omega H_{0}\right) \omega^{\alpha}}{\Gamma(\alpha+1)}+M \sum_{k=1}^{m} M_{k}\right] \alpha(W) \\
& +\left[\frac{2 M L_{1}\left(1+\omega K_{0}+\omega H_{0}\right) \omega^{\alpha}}{\Gamma(\alpha+1)}+M \sum_{k=1}^{m} M_{k}\right] \alpha(W) \\
= & M\left(M M^{*}+1\right)\left[\frac{2 M L_{1}\left(1+\omega K_{0}+\omega H_{0}\right) \omega^{\alpha}}{\Gamma(\alpha+1)}+\sum_{k=1}^{m} M_{k}\right] \alpha(W) .
\end{aligned}
$$

Hence, for any $t \in J$, we have

$$
\alpha\left(Q\left(D_{0}(t)\right)\right) \leq M\left(M M^{*}+1\right)\left[\frac{2 M L_{1}\left(1+\omega K_{0}+\omega H_{0}\right) \omega^{\alpha}}{\Gamma(\alpha+1)}+\sum_{k=1}^{m} M_{k}\right] \alpha(W) .
$$

Since $Q\left(W_{0}\right)$ is bounded and equicontinuous, by Lemma 2.5, (H4) and (H5), we have

$$
\begin{aligned}
\alpha(Q(W)) & \leq 2 \alpha\left(Q\left(W_{0}\right)\right)=2 \max _{t \in J} \alpha\left(Q\left(W_{0}(t)\right)\right) \\
& \leq 2 M\left(M M^{*}+1\right)\left[\frac{2 M L_{1}\left(1+\omega K_{0}+\omega H_{0}\right) \omega^{\alpha}}{\Gamma(\alpha+1)}+\sum_{k=1}^{m} M_{k}\right] \alpha(W)<\alpha(W) .
\end{aligned}
$$

Then $Q: B_{r_{0}}(J) \rightarrow B_{r_{0}}(J)$ is a condensing mapping. By Lemma 2.6, $Q$ has at least one fixed point $u$ in $P C(J, E)$.

Theorem 3.2 Let $E$ be a Banach space, $A: D(A) \subset E \rightarrow E$ be a closed linear operator and $-A$ generate an equicontinuous $C_{0}$-semigroup $T(t)(t \geq 0)$ in $E$. Assume that the set

$$
\begin{aligned}
\left\{R\left(v_{n-1}\right)\right\}= & \left\{B \left[\int_{0}^{\omega}(\omega-s)^{\alpha-1} \mathscr{S}_{\alpha}(\omega-s) f\left(s, v_{n-1}(s), F v_{n-1}(s), G v_{n-1}(s)\right) d s\right.\right. \\
& \left.\left.+\sum_{i=1}^{m} \mathscr{T}_{\alpha}\left(\omega-t_{i}\right) I_{i}\left(v_{n-1}\left(t_{i}\right)\right)\right]: n \in \mathbb{N}\right\}
\end{aligned}
$$

is relatively compact and (H1)-(H3) hold with the following conditions:

(H6) There exist constants $L>0$ such that, for any bounded and equicontinuous sets

$V_{i} \subset C(J, E)(i=1,2,3)$ and $t \in J$,

$$
\beta\left(f\left(t, V_{1}, V_{2}, V_{3}\right)\right) \leq L \sum_{i=1}^{3} \beta\left(V_{i}\right) .
$$


(H7) The inequality

$$
\frac{2 M\left[L+2 \omega L\left(K_{0}+H_{0}\right)\right]}{\Gamma(\alpha+1)} \omega^{\alpha}<1
$$

is satisfied. Then the problem (1.4) has at least one solution on J.

Proof For convenience, we denote $B=\left\{v_{n}: n \in \mathbb{N}\right\}$ and $B_{0}=\left\{v_{n-1}: n \in \mathbb{N}\right\}$. Then $B=Q\left(B_{0}\right)$. We will consider set $B_{0}$ and prove set $B_{0}$ is relatively compact.

It follows from Theorem 3.1 that $B_{0}$ is uniformly bounded and equicontinuous on $J$. Next, we only prove that, for any $t \in J$, set $B_{0}(t)=\left\{v_{n-1}(t): n \in \mathbb{N}\right\}$ is relatively compact in $E$. From $B_{0}=B \cup\left\{v_{0}\right\}$ it follows that $\alpha\left(B_{0}(t)\right)=\alpha(B(t))$ for $t \in J$. Let $\varphi(t):=\alpha(B(t)), t \in J$, going from $J_{0}$ to $J_{m}$ interval by interval we show that $\varphi(t) \equiv 0$ in $J$.

For $t \in J$, there exists a $J_{k-1}$ such that $t \in J_{k-1}$. By (1.5) and Lemma 2.5, we have

$$
\begin{aligned}
\alpha\left(F\left(B_{0}\right)(t)\right)= & \alpha\left(\left\{\int_{0}^{t} K(t, s) v_{n-1}(s) d s: n \in \mathbb{N}\right\}\right) \\
\leq & \sum_{j=1}^{k-1} \alpha\left(\left\{\int_{t_{j-1}}^{t_{j}} K(t, s) v_{n-1}(s) d s: n \in \mathbb{N}\right\}\right) \\
& +\alpha\left(\left\{\int_{t_{k-1}}^{t} K(t, s) v_{n-1}(s) d s: n \in \mathbb{N}\right\}\right) \\
\leq & 2 K_{0} \sum_{j=1}^{k-1} \int_{t_{j-1}}^{t_{j}} \alpha\left(B_{0}(s)\right) d s+2 K_{0} \int_{t_{k-1}}^{t} \alpha\left(B_{0}(s)\right) d s \\
= & 2 K_{0} \sum_{j=1}^{k-1} \int_{t_{j-1}}^{t_{j}} \varphi(s) d s+2 K_{0} \int_{t_{k-1}}^{t} \varphi(s) d s \\
= & 2 K_{0} \int_{0}^{t} \varphi(s) d s,
\end{aligned}
$$

and therefore,

$$
\int_{0}^{t} \alpha\left(F\left(B_{0}\right)(s)\right) d s \leq 2 \omega K_{0} \int_{0}^{t} \varphi(s) d s
$$

and

$$
\begin{aligned}
\alpha\left(G\left(B_{0}\right)(t)\right)= & \alpha\left(\left\{\int_{0}^{\omega} H(t, s) v_{n-1}(s) d s: n \in \mathbb{N}\right\}\right) \\
\leq & \sum_{j=1}^{k-1} \alpha\left(\left\{\int_{t_{j-1}}^{t_{j}} H(t, s) v_{n-1}(s) d s: n \in \mathbb{N}\right\}\right) \\
& +\alpha\left(\left\{\int_{t_{k-1}}^{\omega} H(t, s) v_{n-1}(s) d s: n \in \mathbb{N}\right\}\right) \\
\leq & 2 H_{0} \sum_{j=1}^{k-1} \int_{t_{j-1}}^{t_{j}} \alpha\left(B_{0}(s)\right) d s+2 H_{0} \int_{t_{k-1}}^{\omega} \alpha\left(B_{0}(s)\right) d s
\end{aligned}
$$




$$
\begin{aligned}
& =2 H_{0} \sum_{j=1}^{k-1} \int_{t_{j-1}}^{t_{j}} \varphi(s) d s+2 H_{0} \int_{t_{k-1}}^{\omega} \varphi(s) d s \\
& =2 H_{0} \int_{0}^{\omega} \varphi(s) d s,
\end{aligned}
$$

and therefore,

$$
\int_{0}^{t} \alpha\left(G\left(B_{0}\right)(s)\right) d s \leq 2 \omega H_{0} \int_{0}^{\omega} \varphi(s) d s
$$

For $t \in J_{0}$, from (3.5), using Lemma 2.5, assumption (H6) and (3.10), (3.11), we have

$$
\begin{aligned}
\varphi(t)= & \alpha(B(t))=\alpha\left(Q\left(B_{0}\right)(t)\right) \\
= & \alpha\left(\mathscr{T}_{\alpha}(t) R\left(v_{n-1}\right)+\int_{0}^{t}(t-s)^{\alpha-1} \mathscr{S}_{\alpha}(t-s) f\left(s, v_{n-1}(s), F v_{n-1}(s), G v_{n-1}(s)\right) d s\right) \\
\leq & \frac{2 M}{\Gamma(\alpha)} \int_{0}^{t} \alpha\left(\left\{(t-s)^{\alpha-1}\left(f\left(s, v_{n-1}(s), F v_{n-1}(s), G v_{n-1}(s)\right)\right)\right\}\right) d s \\
\leq & \frac{2 M}{\Gamma(\alpha)} \int_{0}^{t}(t-s)^{\alpha-1}\left(L\left(\alpha\left(B_{0}(s)\right)+\alpha\left(F\left(B_{0}\right)(s)\right)+\alpha\left(G\left(B_{0}\right)(s)\right)\right)\right) d s \\
\leq & \frac{2 M L}{\Gamma(\alpha)} \int_{0}^{t}(t-s)^{\alpha-1} \varphi(s) d s+\frac{4 M}{\Gamma(\alpha)} \omega L K_{0} \int_{0}^{t}(t-s)^{\alpha-1} \varphi(s) d s \\
& +\frac{4 M}{\Gamma(\alpha)} \omega L H_{0} \int_{0}^{\omega}(t-s)^{\alpha-1} \varphi(s) d s .
\end{aligned}
$$

Hence by (H7) and Lemma 3.2, $\varphi(t) \equiv 0$ in $J_{0}$. In particular, $\alpha\left(B\left(t_{1}\right)\right)=\alpha\left(B_{0}\left(t_{1}\right)\right)=\varphi\left(t_{1}\right)=0$, this implies that $B\left(t_{1}\right)$ and $B_{0}\left(t_{1}\right)$ are precompact in $E$. Thus $I_{1}\left(B_{0}\left(t_{1}\right)\right)$ is precompact in $E$, and $\alpha\left(I_{1}\left(B_{0}\left(t_{1}\right)\right)\right)=0$.

Now, for $t \in J_{1}$, by the above argument for $t \in J_{0}$, we have

$$
\begin{aligned}
\varphi(t)= & \alpha(B(t))=\alpha\left(Q\left(B_{0}\right)(t)\right) \\
= & \alpha\left(\left\{\mathscr{T}_{\alpha}(t) R\left(v_{n-1}\right)+\mathscr{T}_{\alpha}\left(t-t_{1}\right) I_{1}\left(v_{n-1}\left(t_{1}\right)\right)\right.\right. \\
& \left.\left.+\int_{0}^{t}(t-s)^{\alpha-1} \mathscr{S}_{\alpha}(t-s) f\left(s, v_{n-1}(s), F v_{n-1}(s), G v_{n-1}(s)\right) d s\right\}\right) \\
\leq & \frac{2 M L}{\Gamma(\alpha)} \int_{0}^{t}(t-s)^{\alpha-1} \varphi(s) d s+\frac{4 M}{\Gamma(\alpha)} \omega L K_{0} \int_{0}^{t}(t-s)^{\alpha-1} \varphi(s) d s \\
& +\frac{4 M}{\Gamma(\alpha)} \omega L H_{0} \int_{0}^{\omega}(t-s)^{\alpha-1} \varphi(s) d s \\
= & \frac{2 M L}{\Gamma(\alpha)} \int_{t_{1}}^{t}(t-s)^{\alpha-1} \varphi(s) d s+\frac{4 M}{\Gamma(\alpha)} \omega L K_{0} \int_{t_{1}}^{t}(t-s)^{\alpha-1} \varphi(s) d s \\
& +\frac{4 M}{\Gamma(\alpha)} \omega L H_{0} \int_{t_{1}}^{\omega}(t-s)^{\alpha-1} \varphi(s) d s .
\end{aligned}
$$

Again by (H7) and Lemma 3.2, $\varphi(t) \equiv 0$ in $J_{1}$, from which we obtain $\alpha\left(B_{0}\left(t_{2}\right)\right)=0$ and $\alpha\left(I_{2}\left(B_{0}\left(t_{2}\right)\right)\right)=0$. 
Continuing such a process interval by interval up to $J_{m}$, we can prove that $\varphi(t) \equiv 0$ in every $J_{k}, k=0,1,2, \ldots, m$. Hence, for any $t \in J, B_{0}(t)$ is relatively compact. Consequently, it follows from the Ascoli-Arzela theorem that set $B_{0}$ is relatively compact, i.e., there exists a convergent subsequence of $\left\{v_{n}\right\}_{n=0}^{\infty}$. With no threat of confusion, $\operatorname{let}_{\lim } \rightarrow \infty v_{n}=v^{*} \in B_{r}(J)$.

Thus, by continuous of the operator $Q$, we have

$$
v^{*}=\lim _{n \rightarrow \infty} v_{n}=\lim _{n \rightarrow \infty} Q v_{n-1}=Q\left(\lim _{n \rightarrow \infty} v_{n-1}\right)=Q v^{*},
$$

which implies the PBVP (1.4) has at least a mild solution. This completes the proof.

\section{Examples}

In this section, we give two examples to demonstrate how to utilize our results.

Example 4.1 We consider the impulsive fractional differential equations with periodic boundary conditions,

$$
\left\{\begin{array}{l}
{ }^{c} D_{0^{+}}^{\frac{1}{2}} u(t, y)+\frac{\partial^{2}}{\partial y^{2}} u(t, y)=\frac{t}{25} \frac{\|u(t, y)\|}{(1+\|u(t, y)\|)}+\frac{1}{5} \int_{0}^{t} \frac{e^{-(s-t)}}{5} u(s, y) d s \\
\quad+\frac{1}{5} \int_{0}^{t} \frac{e^{\frac{-(s-t)}{2}}}{5} u(s, y) d s, \quad y \in(0, \pi), t \in\left[0, \frac{1}{2}\right) \cup\left(\frac{1}{2}, 1\right], \\
\left.\Delta u\right|_{t=\frac{1}{2}}=\frac{\left|u\left(\frac{1^{-}}{2}, y\right)\right|}{\left(20+\left|u\left(\frac{1}{2}, y\right)\right|\right)}, \quad y \in(0, \pi), \\
u(t, 0)=u(t, \pi)=0, \\
u(0, y)=u(1, y), \quad y \in(0, \pi) .
\end{array}\right.
$$

Let $E=L^{2}(0, \pi)$. Define $A u=\frac{\partial^{2}}{\partial y^{2}} u$ for $u \in D(A)$,

$$
D(A)=\left\{u \in E: \frac{\partial u}{\partial y}, \frac{\partial^{2} u}{\partial y^{2}} \in E, u(0)=u(\pi)=0\right\} .
$$

Then $-A$ is the infinitesimal generates of a $C_{0}$-semigroup $T(t)(t \geq 0)$ in $E$, which is equicontinuous and $M=1$. Moreover, $T(\cdot)$ is also compact and $\|T(t)\| \leq e^{-t} \leq 1, t \geq 0$. By the Fredholm alternative theorem, $\left[I-\mathscr{T}_{\alpha}(1)\right]^{-1}$ exists and is bounded where $\mathscr{T}_{\alpha}(\cdot)$ is defined in Section 2.

Let

$$
\begin{aligned}
& F u=\int_{0}^{t} \frac{e^{-(s-t)}}{5} u(s, y) d s, \quad G u=\int_{0}^{t} \frac{e^{\frac{-(s-t)}{2}}}{5} u(s, y) d s, \\
& f(t, u, F u, G u)=\frac{t}{25} \frac{\|u(t, y)\|}{(1+\|u(t, y)\|)}+F u+G u, \quad y \in(0, \pi), t \in\left[0, \frac{1}{2}\right) \cup\left(\frac{1}{2}, 1\right], u \in E
\end{aligned}
$$

and $f:[0,1] \times E \times E \times E \rightarrow E$ is a continuous function.

Next, we can calculate

$$
\begin{aligned}
\|f(t, u, F u, G u)\| & =\left\|\frac{t}{25} \frac{\|u(x, t)\|}{(1+\|u(x, t)\|)}+\frac{1}{5} \int_{0}^{t} \frac{e^{-(s-t)}}{5} u(x, s) d s+\frac{1}{5} \int_{0}^{t} \frac{e^{\frac{-(s-t)}{2}}}{5} u(x, s) d s\right\| \\
& \leq \frac{1}{25}\|u\|+\frac{2}{25}\|u\|+\frac{2}{25}\|u\|=\frac{1}{5}\|u\|,
\end{aligned}
$$

hence the condition (H1) holds with $M_{R}(t)=\frac{1}{5}, \Omega(r)=r$. 
Define

$$
I_{1}\left(u\left(\frac{1}{2}^{-}, y\right)\right)=\frac{\left|u\left(\frac{1}{2}^{-}, y\right)\right|}{\left(20+\left|u\left(\frac{1}{2}^{-}, y\right)\right|\right)}, \quad y \in(0, \pi), u \in E
$$

and

$$
\left\|I_{1}(u)\right\|=\left\|\frac{u}{(20+u)}\right\| \leq \frac{1}{20}\|u\|,
$$

hence the condition (H1) holds with $\rho=\frac{1}{20}$.

Moreover,

$$
M\left(M M^{*}+1\right)\left(\frac{\left\|M_{r_{0}}\right\| \Omega\left(r_{0}\right) \omega^{\alpha}}{(\alpha+1)}+m \rho\right)=\left(M^{*}+1\right)\left(\frac{2 r_{0}}{5 \Gamma\left(\frac{1}{2}\right)}+\frac{1}{20}\right)<r_{0} .
$$

Thus, one can choose $r_{0}>\frac{1}{20} /\left[\frac{1}{M^{*}+1}-\frac{2}{5 \Gamma\left(\frac{1}{2}\right)}\right]$ such that (H3) holds. Furthermore, assume that (H4), (H5) hold. Therefore, by Theorem 3.1, the problem (4.1) has a mild solution on $[0,1]$.

Example 4.2 We consider the impulsive fractional differential equations with periodic boundary conditions,

$$
\left\{\begin{array}{l}
{ }^{c} D_{0^{+}}^{\frac{1}{2}} u(t, y)=\frac{\partial^{2}}{\partial y^{2}} u(t, y)+\frac{\|u(t, y)\|}{\left(1+9 e^{t}\right)(1+\|u(t, y)\|)}+\int_{0}^{t} \frac{e^{-(s-t)}}{10} u(s, y) \sin u(s, y) d s \\
\quad+\int_{0}^{t} \frac{e^{\frac{-(s-t)}{2}}}{10} u(s, y) \cos u(s, y) d s, \quad y \in(0, \pi), t \in\left[0, \frac{1}{2}\right) \cup\left(\frac{1}{2}, 1\right], \\
\left.\Delta u\right|_{t=\frac{1}{2}}=\frac{\left|u\left(\frac{1}{2}^{-}, y\right)\right|}{(9+t)\left(1+\left|u\left(\frac{1}{2}^{-}, y\right)\right|\right)}, \quad y \in(0, \pi), \\
u(t, 0)=u(t, \pi)=0, \\
u(0, y)=u(1, y), \quad y \in(0, \pi) .
\end{array}\right.
$$

Let $E=L^{2}(0, \pi)$. Define $A u=-\frac{\partial^{2}}{\partial y^{2}} u$ for $u \in D(A)$, where

$$
D(A)=\left\{u \in E: \frac{\partial u}{\partial y}, \frac{\partial^{2} u}{\partial y^{2}} \in E, u(0)=u(\pi)=0\right\} .
$$

Then $A$ is the infinitesimal generates of a $C_{0}$-semigroup $T(t)(t \geq 0)$ in $E$, which is equicontinuous and $M=1$. Moreover, $T(\cdot)$ is also compact and $\|T(t)\| \leq e^{-t} \leq 1, t \geq 0$. By the Fredholm alternative theorem, $\left[I-\mathscr{T}_{\alpha}(1)\right]^{-1}$ exists and is bounded where $\mathscr{T}_{\alpha}(\cdot)$ is defined in Section 2.

Let

$$
\begin{aligned}
& F u=\int_{0}^{t} \frac{e^{-(s-t)}}{10} u(s, y) \sin u(s, y) d s, \quad G u=\int_{0}^{t} \frac{e^{\frac{-(s-t)}{2}}}{10} u(s, y) \cos u(s, y) d s, \\
& f(t, u, F u, G u)=\frac{\|u(t, y)\|}{\left(1+9 e^{t}\right)(1+\|u(t, y)\|)}+F u+G u, \\
& y \in(0, \pi), t \in\left[0, \frac{1}{2}\right) \cup\left(\frac{1}{2}, 1\right], u \in E,
\end{aligned}
$$

and $f:[0,1] \times E \times E \times E \rightarrow E$ is a continuous function. 
Next, we can calculate

$$
\begin{aligned}
\|f(t, u, F u, G u)\|= & \| \frac{\|u(t, y)\|}{\left(1+9 e^{t}\right)(1+\|u(t, y)\|)}+\int_{0}^{t} \frac{e^{-(s-t)}}{10} u(s, y) \sin u(s, y) d s \\
& +\int_{0}^{t} \frac{e^{\frac{-(s-t)}{2}}}{10} u(s, y) \cos u(s, y) d s \| \\
\leq & \frac{1}{10}\|u\|+\frac{2}{10}\|u\|+\frac{2}{10}\|u\|=\frac{1}{2}\|u\|,
\end{aligned}
$$

hence the condition (H1) holds with $M_{R}(t)=\frac{1}{2}, \Omega(r)=r$.

Define

$$
I_{1}\left(u\left(\frac{1}{2}^{-}, y\right)\right)=\frac{\left|u\left(\frac{1}{2}^{-}, y\right)\right|}{(9+t)\left(1+\left|u\left(\frac{1}{2}^{-}, y\right)\right|\right)}, \quad y \in(0, \pi), u \in E
$$

hence the condition (H1) holds with $\rho=\frac{1}{10}$.

Moreover,

$$
M\left(M M^{*}+1\right)\left(\frac{\left\|M_{r_{0}}\right\| \Omega\left(r_{0}\right) \omega^{\alpha}}{\Gamma(\alpha+1)}+m \rho\right)=\left(M^{*}+1\right)\left(\frac{r_{0}}{\Gamma\left(\frac{1}{2}\right)}+\frac{1}{10}\right)<r_{0} .
$$

Thus, one can choose $r_{0}>\frac{1}{10} /\left[\frac{1}{M^{*}+1}-\frac{1}{\Gamma\left(\frac{1}{2}\right)}\right]$ such that (H3) holds. Furthermore, assume that (H4), (H5) holds. Therefore, by Theorem 3.1, the problem (4.2) has a mild solution on $[0,1]$.

\section{Conclusions}

In this paper, by applying some well-known fixed point theorem, many new existence theorems of solutions are established under weaker conditions. In particular, we can extend the well-known results in $\mathrm{Mu}[29,30]$ and point out some essential flaws in their manuscripts.

\section{Acknowledgements}

The authors wish to thank the referees for their valuable comments. We would also like to acknowledge the valuable comments and suggestions from the reviewers and editors, which vastly contributed to improve the presentation of the paper. This work is supported by National Natural Science Foundation of China (11061031).

\section{Competing interests}

The authors declare that they have no competing interests.

\section{Authors' contributions}

All authors contributed equally to the writing of this paper. All authors read and approved the final manuscript.

\section{Publisher's Note}

Springer Nature remains neutral with regard to jurisdictional claims in published maps and institutional affiliations.

Received: 24 March 2017 Accepted: 30 June 2017 Published online: 15 August 2017

\section{References}

1. Benchohra, M, Henderson, J, Ntouyas, S: Impulsive Differential Equations and Inclusions. Contemporary Mathematics and Its Applications, vol. 2. Hindawi Publ. Corp., New York (2006)

2. Agarwal, RP, Benchohra, M, Hamani, S: A survey on existence result for boundary value problem of nonlinear fractional differential equations and inclusions. Acta Appl. Math. 109, 973-1033 (2010)

3. Ahmad, B, Sivasundaram, S: Existence results for nonlinear impulsive hybrid boundary value problems involving fractional differential equations. Nonlinear Anal. Hybrid Syst. 3, 251-258 (2009)

4. Benchohra, M, Seba, D: Impulsive fractional differential equations in Banach spaces. Electron. J. Qual. Theory Differ. Equ. 8, 1 (2009) 
5. Balachandran, K, Kiruthika, S: Existence of solutions of abstract fractional impulsive semilinear evolution equations. Electron. J. Qual. Theory Differ. Equ. 4, 1 (2010)

6. Wang, G, Zhang, L, Song, G: Systems of first order impulsive functional differential equations with deviating arguments and nonlinear boundary conditions. Nonlinear Anal. TMA 74, 974-982 (2011)

7. Wang, JR, Zhou, Y, Fečkan, M: On recent developments in the theory of boundary value problems for impulsive fractional differentail equations. Comput. Math. Appl. 64, 3008-3020 (2012)

8. Wang, JR, Fečkan, M, Zhou, Y: Ulam's type stability of impulsive ordinary differential equations. J. Math. Anal. Appl. 395, 258-264 (2012)

9. Wang, JR, Li, X, Wei, W: On the natural solution of an impulsive fractional differential equation of order $q \in(1,2)$. Commun. Nonlinear Sci. Numer. Simul. 17, 4384-4394 (2012)

10. Fečkan, $M, Z$ hou, $Y$, Wang, JR: On the concept and existence of solution for impulsive fractional differential equations. Commun. Nonlinear Sci. Numer. Simul. 17, 3050-3060 (2012)

11. Wang, G, Ahmad, B, Zhang, L, Nieto, JJ: Comments on the concept of existence of solution for impulsive fractional differential equations. Commun. Nonlinear Sci. Numer. Simul. 19, 401-403 (2014)

12. Wang, J, Fečkan, M, Zhou, Y: On the new concept of solutions and existence results for impulsive fractional evolution equations. Dyn. Partial Differ. Equ. 8, 345-361 (2011)

13. Wang, J, Zhou, Y, Fečkan, M: Abstract Cauchy problem for fractional differential equations. Nonlinear Dyn. 74, 685-700 (2013)

14. Shu, XB, Shi, YJ: A study on the mild solution of impulsive fractional evolution equations. Appl. Comput. Math. 273, 465-476 (2016)

15. Wang, J, Li, XZ: Ulam-Hyers stability of fractional Langevin equations. Appl. Math. Comput. 258, 72-83 (2015)

16. Wang, J, Li, XZ: A uniform method to Ulam-Hyers stability for some linear fractional equations. Mediterr. J. Math. 13, 625-635 (2016)

17. Wang, J, Fečkan, M, Zhou, Y: A survey on impulsive fractional differential equations. Fract. Calc. Appl. Anal. 19, 806-831 (2016)

18. Wang, J, Zhou, Y, Fečkan, M: Nonlinear impulsive problems for fractional differential equations and Ulam stability. Comput. Math. Appl. 64, 3389-3405 (2012)

19. Wang, J, Fečkan, M, Zhou, Y: Relaxed controls for nonlinear frational impulsive evolution equations. J. Optim. Theory Appl. 156, 13-32 (2013)

20. Liu, Z, Li, XW: On the controllability of impulsive fractional evolution inclusions in Banach spaces. J. Optim. Theory Appl. 156, 167-182 (2013)

21. Fečkan, M, Zhou, Y, Wang, J: Response to comments on the concept of existence of solution for impulsive fractional differential equations [Commun Nonlinear Sci Numer Simulat. 19, 401-403 (2014)]. Commun. Nonlinear Sci. Numer. Simul. 19, 4213-4215 (2014)

22. Ye, $\mathrm{H}, \mathrm{Gao}, \mathrm{J}$, Ding, Y: A generalized Gronwall inequality and its applications to a fractional differential equation. J. Math. Anal. Appl. 328, 1075-1081 (2007)

23. Wang, J, Zhou, Y, Fečkan, M: Alternative results and robustness for fractional evolution equations with periodic boundary conditions. Electron. J. Qual. Theory Differ. Equ. 97, 1 (2011)

24. Liu, YJ, Ahmad, B: A study of impulsive multiterm fractional differential equations with single and mutiple base point and applications. Sci. World J. 2014 Article ID 194346 (2014)

25. Xie, SL: Existence results of mild solutions for impulsive fractional integro-differential evolutions with infinite delay. Fract. Calc. Appl. Anal. 17, 1158-1174 (2014)

26. Chen, $\mathrm{PY}, \mathrm{Li}, \mathrm{YX}, \mathrm{Chen}, \mathrm{QY}$, Feng, BH: On the initial value problem of fractional evolution equations with noncompact semigroup. Comput. Math. Appl. 67, 1108-1115 (2014)

27. Yu, XL, Wang, JR: Periodic BVPs for fractional order impulsive evolution equations. Bound. Value Probl. 201435 (2014)

28. Shu, XB, Xu, F, Shi, Y: S-asymptotically $\omega$-positive periodic solutions for a class of neutral fractional differential equations. Appl. Comput. Math. 270, 768-776 (2015)

29. $\mathrm{Mu}, \mathrm{J}, \mathrm{Li}, \mathrm{YX}$ : Monotone interactive technique for impulsive fractional evolution equations. J. Inequal. Appl. 2011, 125 (2011)

30. $\mathrm{Mu}, \mathrm{J}$ : Extremal mild solutions for impulsive fractional evolution equations with nonlocal initial conditions. Bound. Value Probl. 201271 (2012)

31. Li, YX: The positive solutions of abstract semilinear evolution equations and their applications. Acta Math. Sin. 39(5), 666-672 (1996) (in Chinese)

32. Guo, DJ, Sun, JX: Ordinary Differential Equations in Abstract Spaces. Shandong Science and Technology, Ji'nan (1989) (in Chinese)

33. Heinz, HR: On the behavior of measure of noncompactness with respect to differentiation and integration of vector-valued functions. Nonlinear Anal. 71, 1351-1371 (1983) 